\title{
Properties and Cost Effective Method for Production of the Antitumor Agent Duclauxin from Sporulating Penicillium herquei
}

\author{
Frank O. Bryant, horace G. Cutler ${ }^{x}$, and John M. Jacyno
}

Received March 25, 1992, from USDA, Agricultural Research Service, P.O. Box 5677, Athens, GA $30613 . \quad$ Accepted for publication February 25, 1993.

\begin{abstract}
Duclauxin, an antitumor agent, was isolated from sporulating Penicillium herquei (ATCC 34665) grown on a medium of peanut hulls supplemented with potato starch solution (termed "Gostar"). The medium was inoculated with a sporulating subculture of $P$. herquei established on a $2 \%$ potato starch slurry supplemented with mineral salts. The $P$. herquei grew as well on Gostar as on an enriched medium. Duclauxin was isolated in crystalline form from Gostar-grown $P$. herquei. Comparison of costs of duclauxin obtained from inexpensive Gostar versus costly enriched media indicated that Gostar reduces production expenses. Duclauxin was not effective as an antibiotic against certain species of gram-positive and gram-negative bacteria, fungi, and viruses, but a concentration-dependent inhibition of wheat coleoptile growth was observed. Duclauxin was characterized by melting point, optical rotation, IR and NMR spectroscopy, MS and X-ray diffraction.
\end{abstract}

Duclauxin is an excellent prospect as an antitumor agent. Chemically, it consists of a heptacyclic system, containing an isocoumarin and a dihydroisocoumarin nucleus. ${ }^{1,2}$ Duclauxin is produced by Penicillium duclauxii, ${ }^{1} P$. stipitatum,$^{3}$ and $P$. herquei.4,5 Duclauxin has been shown to be effective against Ehrlich's ascites carcinoma cells, ${ }^{3,6-9}$ Lymphadenoma L-5178, ${ }^{3}$ HeLa cells, ${ }^{3}$ tumor cells of the line P $388,{ }^{10}$ and murine leukemia L1210 culture cells. ${ }^{8,9,11}$ The mode of action of duclauxin involves inhibition of mitochondrial respiration (uncoupling of oxidative phosphorylation). ${ }^{8-11}$

Among the problems associated with isolating naturally occurring antitumor agents are the low yields and high production costs. To reduce production costs, we developed a method of culturing $P$. herquei in which peanut hulls and potato starch serve as substrate. This medium is termed "Gostar". Equally important is the potential for culturing other fungi on a Gostar-type medium when liquid culture is not possible, leading to the production of valuable products. This may be fruitful because numerous fungal species have been isolated from or associated with peanut hulls. ${ }^{12}$

We now describe the growth of $P$. herquei on Gostar

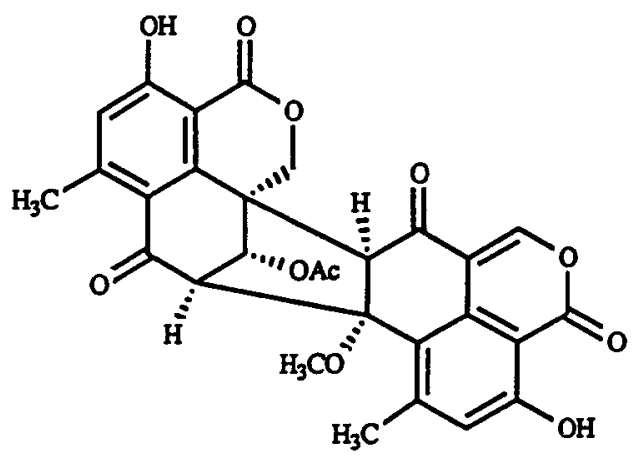

Duclauxin medium, the first reported isolation of crystalline duclauxin from $P$. herquei, antibiotic resistance testing, and other physical characteristics of duclauxin.

\section{Experimental Section}

Sporulation of $P$. herquei for Inoculum-A lyophilized culture of $P$. herquei (ATCC 34665) was rehydrated and transferred to $20-\mathrm{mL}$ screw-capped tubes containing $10 \mathrm{~mL}$ of potato dextrose agar (PDA, Difco) slants and grown for 7 days until mycelia covered the surface. The fungus was transferred from the PDA medium to $20-\mathrm{mL}$ screwcapped tubes containing $10 \mathrm{~mL}$ of liquid medium consisting of $2 \%$ potato starch, $0.2 \% \mathrm{NaNO}_{3}, 0.1 \% \mathrm{KH}_{2} \mathrm{PO}_{4}, 0.5 \% \mathrm{KCl}, 0.05 \%$ $\mathrm{MgSO}_{4} \cdot 7 \mathrm{H}_{2} \mathrm{O}$, and $0.001 \% \mathrm{FeSO}_{4} \cdot 7 \mathrm{H}_{2} \mathrm{O}(\mathrm{PSM})$; the final $\mathrm{pH}$ of this solution was $\mathrm{pH}$ 6.3. The PSM was autoclaved at $121^{\circ} \mathrm{C}$ for $25 \mathrm{~min}$. No further adjustment of $\mathrm{pH}$ was required. Inoculated tubes were incubated as stationary cultures at $29^{\circ} \mathrm{C}$, and sporulation was monitored by direct cell count with an Improved Neubauer chamber $(1 / 400 \mathrm{sq} . \mathrm{mm} \times 1 / 10 \mathrm{~mm})$. All manipulations were performed with sterile conditions.

Gostar Medium-Gostar medium was prepared in 14 2.8-L Fernbach flasks containing dry peanut hulls $\sim 5 \mathrm{~cm}$ in depth $(-500 \mathrm{~mL}$ loose peanut hulls). A $2 \%$ aqueous slurry of potato starch was prepared, and $200 \mathrm{~mL}$ were added to each Fernbach flask. Flasks were covered with inverted $500 \mathrm{~mL}-\mathrm{Nalgene}$ beakers containing cotton $\sim 2$ cm deep and autoclaved at $121^{\circ} \mathrm{C}$ for $45 \mathrm{~min}$; the flasks were then cooled at room temperature overnight. This procedure resulted in the potato starch coating the peanut hulls. Each flask containing Gostar medium was inoculated with $\sim 1 \mathrm{~mL}$ of $P$. herquei spores subcultured successively through the third generation $\left(G_{3}\right)$ and incubated at $23{ }^{\circ} \mathrm{C}$ $( \pm 2)$ in the light. $P$. herquei was incubated at $23^{\circ} \mathrm{C}( \pm 2)$ for $2-4$ weeks, with harvesting at regular intervals.

Extraction of Duclauxin from $P$. herquei Grown on Gostar Medium-All chemicals and solvents were reagent grade. Growth of $P$. herquei was terminated by the addition of sufficient acetone $(-500$ $\mathrm{mL}$ ) to cover the Gostar medium in each Fernbach flask. Groups of three Fernbach flasks were combined and homogenized with an Ultra-Turrax apparatus (Janke and Kunkel KG, Breisgau, Germany). This homogenate was vacuum-filtered through Whatman \#4 filter paper $(24 \mathrm{~cm})$ in a Büchner funnel. The filtrate was collected and concentrated under reduced pressure at $55^{\circ} \mathrm{C}$ to $-500 \mathrm{~mL}$, then extracted twice with an equal volume of ethyl acetate in a separatory flask. The ethyl acetate phase (upper fraction), which contained the duclauxin, was dried over granular, anhydrous $\mathrm{Na}_{2} \mathrm{SO}_{4}$ overnight, then passed through a glass column $(10 \times 10 \mathrm{~cm})$ containing anhydrous $\mathrm{Na}_{2} \mathrm{SO}_{4}$ and evaporated to dryness under reduced pressure. The dried residue was dissolved in $-5 \mathrm{~mL}$ of ethyl acetate, and this solution was applied to a column $(9 \times 20 \mathrm{~cm})$ of Silica Gel 60 (70-230 mesh, Merck) that was slurry-packed in benzene. The column was eluted in a stepwise manner with $1 \mathrm{~L}$ each of benzene, ethyl acetate, and acetone, and the fractions were collected individually from each solvent elution. Bulk fractions were evaporated separately to dryness under reduced pressure, and residues were dissolved in $\sim 5$ $\mathrm{mL}$ of ethyl acetate. Fractions containing duclauxin were identified as described (vide infra) and all that contained duclauxin were pooled. TLC was performed to evaluate chromatographic fractions for the presence of duclauxin. Silica Gel $60 \mathrm{~F}_{254}(5 \times 10 \mathrm{~cm}, 0.25-\mathrm{mm}$ thick $)$ TLC plates (Merck) were spotted at the origin with $1 \mu \mathrm{L}$ of sample and developed with toluene:ethyl acetate:formic acid/(5:4:1) as the mo- 
bile phase. The mobile phase was allowed to ascend to $1.5 \mathrm{~cm}$ from the top of the TLC plate before removing the TLC plate from the chamber. Plates were dried, then sprayed with $0.5 \%$ anisaldehyde solution dissolved in $10 \mathrm{~mL}$ of glacial acetic acid, $85 \mathrm{~mL}$ of methanol, and $5 \mathrm{~mL}$ of concentrated sulfuric acid. ${ }^{13}$ Developed plates were heated at $105{ }^{\circ} \mathrm{C}$ for $20 \mathrm{~min}$ to visualize chromatographed compounds. Previously purified duclauxin from $P$. herquei $(1 \mathrm{mg} / \mathrm{mL})$ was used as the internal standard.4

The pooled fractions were chromatographed in an open glass column $(3 \times 45 \mathrm{~cm})$ of Silica Gel $60(70-230$ mesh) equilibrated with benzene. The column was washed with $-500 \mathrm{~mL}$ of benzene, and then a linear gradient of benzene to ethyl acetate was applied $1 \mathrm{~L}$ each reservoir). Fractions of $16 \mathrm{~mL}$ were collected, and every third fraction was analyzed by TLC to determine the presence of duclauxin. Those fractions containing duclauxin were pooled and concentrated. The concentrated fractions were applied to a column $(1.75 \times 25 \mathrm{~cm})$ of Silica Gel 60 equilibrated with methylene chloride : acetone $(9: 1, \mathrm{v} / \mathrm{v})$. Duclauxin eluted with the equilibrating solvent and was concentrated to dryness under reduced pressure. The residue was dissolved in a minimum volume of ethyl acetate and stored at $4^{\circ} \mathrm{C}$. Duclauxin crystals formed within $24 \mathrm{~h}$. The supernatant solution was decanted, and the crystals were redissolved in ethyl acetate and recrystallized to remove impurities. Recrystallization was repeated until only duclauxin remained. Recrystallization of duclauxin in decanted supernatant solutions followed until virtually all the duclauxin was recovered.

Bioassays-Etiolated wheat coleoptiles were used to determine stimulatory or inhibitory biological activity as previously described.14 Microbial bioassays utilized gram-positive and gramnegative bacteria and fungi as previously described. ${ }^{14}$ Antiviral activity was performed in light and in dark against Mouse CMV and Sindbis viruses as previously described. ${ }^{15}$

Physical Analyses-The melting point (mp) was measured on a Kofler block (hot-stage microscope apparatus) and is uncorrected. The IR spectrum was obtained from a KBr disc with an Analect FX-6160 FTIR spectrometer. ${ }^{1} \mathrm{H}$ and ${ }^{13} \mathrm{C}$ NMR spectra were recorded from $\mathrm{CDCl}_{3}$ solutions on a Bruker AM-400 spectrometer. Optical rotation was performed with a Perkin Elmer model 141 polarimeter with $\mathrm{CHCl}_{3}$ as the solvent blank. MS was performed with a HewlettPackard 5985 B mass spectrometer interfaced with a computer programed with the standard reference data base of the National Institute of Standards and References. Samples were injected into the mass spectrometer ballistically at a temperature of $170^{\circ} \mathrm{C}$. X-ray diffraction was performed by mounting crystals in a random orientation on a glass fiber for analysis with a Enraf-Nonius CAD-4 diffractometer with $\mathrm{CuK} \alpha$ radiation at ambient temperature.

\section{Results}

Growth of $P$. herquei and Isolation of Duclauxin-An inoculum of sporulating $P$. herquei was prepared by growing the organism in a $2 \%$ potato starch slurry supplemented with a mineral salts solution. Selected potato starch concentrations from 0.5 to $9 \%$ were tested, but $2 \%$ was found to be optimal for the highest induction of spores. At concentrations of potato starch of $1 \%$ or less, mycelia and fruiting bodies were observed, but as the concentration of potato starch was increased beyond $2 \%$, the number of spores decreased. Figure 1 shows sporulation cycles for three successive subcultures of $P$. herquei grown on liquid PSM. P. herquei spore counts for $G_{3}$ were higher than for the first $\left(G_{1}\right)$ or second $\left(G_{2}\right)$ generations. Consequently, $G_{3}$ was selected as inoculum for the Gostar medium based on its apparent vitality. Also, subculturing $P$. herquei through the third generation on PSM resulted in an inoculum that approached $100 \%$ spores. Because duclauxin is a secondary metabolite and because inoculation of enriched medium with $P$. herquei not subcultured for spore production resulted in comparatively lower yields of duclauxin (data not shown), the efficacy of subculturing spores is apparent. However, a detailed study of the nature of the subculturing has not been performed.

Optimal conditions for sporulation of $P$. herquei were determined by the rate of spore formation. Sporulation was best at $27-29^{\circ} \mathrm{C}$, in screw-capped culture tubes compared

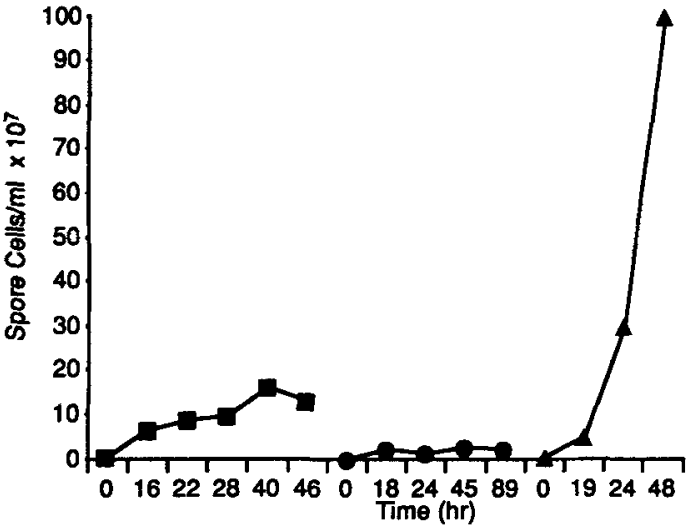

Figure 1-Average spore numbers of $P$. herquei transferred through three generations of growth. Inoculation was with $0.1 \%$ of the previous generation. Key: $(\square) G_{1} ;(\Theta) G_{2} ;(\Delta) G_{3}$.

with sponge-capped or to $\mathrm{N}_{2}$-flushed, stoppered culture tubes. Spore cultures were more prolific in still than shake cultures. For each variable, the observation accounts only for spore concentration in the culture (mycelia were not counted). These favorable conditions were used during growth of each generation of $P$. herquei and may have aided in spore induction. Attempts to induce sporulation of $P$. herquei with corn starch or corn steep liquor, as opposed to potato starch, were not successful.

Gostar medium promoted visible growth of $P$. herquei that was indistinguishable from the enriched medium previously described. ${ }^{16}$ Flasks were inoculated with $1 \mathrm{~mL}$ of $\mathrm{G}_{3}$ spores of $P$. herquei (Figure 1). A thick, green fungal mat covered the peanut hulls, and mycelia extended into the liquid in the bottom of the flask exactly as when grown on enriched medium.

Duclauxin was isolated in crystalline form by the extraction procedure described followed by column chromatography. The purification was monitored by TLC analysis. Those fractions containing duclauxin were pooled and concentrated prior to further purification. By the final stage of purification, a mixture of duclauxin and three other compounds remained. On standing overnight at $4{ }^{\circ} \mathrm{C}$, colorless crystals formed from an amber supernatant solution. TLC analysis indicated the presence of duclauxin and the three other metabolites in the supernatant solution. Duclauxin was the only compound in the crystals as determined by cochromatography with duclauxin previously isolated.

Bioassays-Certain bacterial species including Bacillus subtilis, B. cereus, Microbacterium thermosphactum, Escherichia coli, Enterobacter cloacae, and Citrobacter freundii were resistant to duclauxin as determined with standard PDA plate growth. Certain fungi, including Curvularia lunata, Gaeumannomyces graminis, and Aspergillus flavus, were also resistant to duclauxin. Duclauxin was found not to have antiviral activity, either in light or in dark, against Mouse CMV or Sindbis viruses. By contrast, duclauxin was found to significantly inhibit etiolated wheat coleoptiles $(p<0.01) .17$ The percents of inhibition at selected concentrations of duclauxin relative to controls were: $100 \%$ at $10^{-3} \mathrm{M}, 80 \%$ at $10^{-4} \mathrm{M}, 39 \%$ at $10^{-5} \mathrm{M}$, and $0 \%$ at $10^{-6} \mathrm{M}$.

Physical Characteristics-The mp was $196-198^{\circ} \mathrm{C}$, with softening at $194^{\circ} \mathrm{C}$ (lit. $\left.\mathrm{mp} 230^{\circ} \mathrm{C}^{1} ; 235-236{ }^{\circ} \mathrm{C}^{16}\right)$. The optical rotation value $([\alpha] \mathrm{D})$ of a $1 \%$ solution of duclauxin in $\mathrm{CHCl}_{3}$ was + 164.5 (lit $[\alpha] \mathrm{D}+272.516$ ); $\mathrm{IR}$ (KBr disc): 3300-2850 (w, br) 1751, 1703, 1682, 1629, 1567, 1280, 1228, 1200, 1077, and $757 \mathrm{~cm}^{-1}$ (consistent with the literature ${ }^{1}$ ); ${ }^{1} \mathrm{H} \mathrm{NMR}\left(\mathrm{CDCl}_{3}\right.$ ): $\delta 11.70(\mathrm{~s}, 1 \mathrm{H}), 10.66(\mathrm{~s}, 1 \mathrm{H}), 7.70(\mathrm{~s}, 1 \mathrm{H}), 6.90(\mathrm{~s}, 1 \mathrm{H}), 6.64(\mathrm{~s}$, $1 \mathrm{H}), 5.20(\mathrm{~s}, 1 \mathrm{H}), 5.08(\mathrm{~d}, J=12 \mathrm{~Hz}, 1 \mathrm{H}), 4.78(\mathrm{~d}, J=12 \mathrm{~Hz}$, 
1H), 4.14 (s, 1H), 3.98 (s, 1H), 2.97 (s, 3H), 2.74 (s, 3H), 2.22 $(\mathrm{s}, 3 \mathrm{H}), 2.11(\mathrm{~s}, 3 \mathrm{H})$ (virtually identical to that reported in the literature $\left.{ }^{16}\right) ;{ }^{13} \mathrm{C} \mathrm{NMR}\left(\mathrm{CDCl}_{3}\right): 193.65,190.80,169.47$, $167.25,164.75,163.80,161.74,151.97,148.68,142.83,132.85$, $121.32,120.90,120.78,118.21,113.21,104.77,101.36,88.72$, 78.82, 71.32, 67.33, 63.98, 51.76, 51.06, 22.58, 22.15, 21.03, and $20.92 \mathrm{ppm}$.

MS analysis indicated a molecular ion peak $(\mathbf{M}+)$ at $\mathrm{m} / z$ of 546 with specific ion peaks at $m / z$ of 259,85 , and 83 . No other peaks were detected. The molecular formula was calculated to be $\mathrm{C}_{29} \mathrm{H}_{22} \mathrm{O}_{11}$. This result is in exact agreement with the molecular weight (546.49) and formula of duclauxin. Specific ion peaks corresponded to the molecular weights of tricyclic rings (259) and aromatic rings (85 and 83) of duclauxin.

X-ray diffraction was performed with single crystals of duclauxin grown from ethyl acetate that were colorless thin plates of dimensions $0.05 \times 0.4 \times 0.5 \mathrm{~mm}$. Twenty five diffraction maxima between $\Theta 15-30^{\circ}$ produced an orientation matrix with cell dimensions $(\AA)$ of $a=13.179(1), b=$ $12.074(1), c=17.963(1)$, and $\beta=109.10(7)^{\circ}$, and a cell volume of $2701.0(5) \AA^{3}$. Systematic absences along the unique $b$ axis of the monoclinic cell indicated a space group of $P 2_{1}$. With a formula weight of 546.49 amu and four molecules per cell, a reasonable density of $1.34 \mathrm{~g} / \mathrm{cm}^{3}$ was calculated. Therefore, there are two molecules per asymmetric unit, and a solution of the structure requires determination of 80 nonhydrogen atomic positions.

\section{Discussion}

Of particular importance is the cost of production of duclauxin from $P$. herquei grown on Gostar medium compared to enriched media. Table I compares costs based on current media prices. Although the yields of duclauxin from the Gostar-type media (7.71-1.32 mg/flask) are less than for the enriched medium ( $8.57 \mathrm{mg} /$ flask), the cost comparison greatly favors the Gostar-type medium for this process. Replacing the shredded wheat of the enriched medium with peanut hulls accounts for much of the cost savings. Lowering the yeast extract and mycological broth to $25 \%$ of that in the enriched medium further reduces the cost of duclauxin but does not significantly reduce the yield. Because $P$. herque $i$ was isolated from green peanuts ${ }^{4}$ and because peanut hulls and potato starch effluent are, respectively, a liability ${ }^{18}$ and a waste, ${ }^{19}$ these substrates are not only inexpensive but are suitable for the production of a valuable and costly pharmaceutical produced by the fungus. Alternate uses of peanut hulls as fuel for boilers, mulch, roughage for cattle feed, litter for poultry houses, compressed logs, etc. are of limited demand given the irregular supply. ${ }^{18,20}$ By contrast, the yearly requirement for

Table I-Media Cost Comparison of Duclauxin Production by P. herquel Grown on Gostar versus Enriched Medium

\begin{tabular}{lccc}
\hline \multicolumn{1}{c}{ Medium } & $\begin{array}{c}\text { Mg duclauxin// } \\
\text { Fembach } \\
\text { Flask }\end{array}$ & $\begin{array}{c}\text { Cost/ } \\
\text { Fermbach } \\
\text { Flask, } \$^{b}\end{array}$ & $\begin{array}{c}\text { Cost/ } \\
100 ~ \mathrm{mg} \\
\text { duclauxin, } \$^{b}\end{array}$ \\
\hline $\begin{array}{l}\text { Enriched medium (shredded } \\
\text { wheat, sucrose 20\%, YX }\end{array}$ & 8.57 & 2.43 & 28.35 \\
2\%, MB 5\%) & & & \\
Gostar + YX 0.5\%, MB 1.25\% & 7.71 & 0.36 & 4.67 \\
Gostar + YX 0.25\%, MB 0.5\% & 2.91 & 0.21 & 7.22 \\
Gostar & 1.32 & 0.05 & 3.79 \\
Peanut hulls only (minus & 1.57 & 0.00 & -0.00 \\
starch) & 0 & $-{ }^{c}$ & - \\
Liquid - 2\% potato starch & & & \\
with 0.25\% YX & & & \\
\hline
\end{tabular}

${ }^{a}$ YX: yeast extract (Difco); MB: mycological broth (Difco). ${ }^{b}$ All costs are in US dollars and based on current prices of $Y X$ and MB of $\sim \$ 36.00$ per 454 g. ${ }^{c} \ldots$, Not applicable. peanut hulls as media would be minimal compared with the estimated 125000 tons of hulls that a single processing plant may accumulate per year. ${ }^{18}$ Similarly, the effluent from potato processing plants contains considerable starch that is not considered economically feasible to recover but that could serve as fermentation substrate, based on composition, without further treatment. ${ }^{21}$ Presently, most effluent is treated as a waste product requiring biological oxidation or land disposal. 21

Bacterial resistance testing indicated that duclauxin does not have antibacterial properties against those gram-positive and gram-negative organisms tested. This is not surprising because the apparent mode of action of duclauxin is inhibition of mitochondrial oxidative phosphorylation that does not exist in bacteria. Duclauxin also displayed no antifungal properties against those species tested. Because fungal metabolism may proceed by glycolysis or membrane-bound electron transport mechanisms, inhibition of mitochondrial oxidative phosphorylation would not necessarily be antibiotic. As expected, duclauxin displayed no antiviral activity. However, duclauxin displayed a concentration-dependent inhibition of growth of etiolated wheat coleoptiles that are sensitive to plant growth regulators. ${ }^{22}$ Because duclauxin displayed no antibiotic activity toward normally growing bacteria, fungi, and viruses of this study and because malignant tumor cells (like etiolated coleoptiles) are characterized by rapid cell growth and division, duclauxin apparently displays selectivity in targeting tumor cells.

Duclauxin obtained from $P$. herquei grown on either Gostar or enriched medium displayed $\mathrm{mp}\left(196-198^{\circ} \mathrm{C}\right)$ and optical rotation $([\alpha] \mathrm{D}+164.5)$ values that were lower than those reported by previous researchers $\left(230^{\circ} \mathrm{C}^{1}\right.$ and $235-236^{\circ} \mathrm{C}, 16$ and $\left.[\alpha] D+272.5^{1}\right)$. However, IR and ${ }^{1} \mathrm{H}$ NMR spectra were essentially identical to those previously reported.1,16 To insure that the compound isolated in this study was duclauxin, MS analysis was performed. The result indicated the exact molecular weight (546) and molecular formula $\left(\mathrm{C}_{29} \mathrm{H}_{22} \mathrm{O}_{11}\right)$ expected for duclauxin. Also, specific ion peaks were consistent with molecular weights of the tricyclic rings (259) and the aromatic rings ( 85 and 83 ) that would be expected for duclauxin. Further, X-ray diffraction indicated no discrepancies with the known structure of duclauxin. The unit cell is composed of four molecules of duclauxin, of which two molecules are symmetrical and two are asymmetrical. Preliminary structural analysis also indicated that a single molecule of ethyl acetate is associated with each molecule of duclauxin.

Because duclauxin in this study was crystallized from ethyl acetate and the duclauxin from previous studies was crystallized from benzene or acetone/ethanol, the ethyl acetate trapped in the crystal lattice may account for the observed differences in $\mathrm{mp}$ and optical rotation. Structural analysis by $\mathrm{X}$-ray diffraction of monobromoduclauxin crystallized from acetone/ethanol indicated no residual solvent contained in the crystalline lattice. ${ }^{2}$ Attempts to recrystallize duclauxin in benzene (ethanol and toluene were not successful) resulted in a somewhat higher observed $\mathrm{mp}\left(\sim 216^{\circ} \mathrm{C}\right)$, although not as high as previously reported. Because duclauxin is composed of two tricyclic rings hinged by a five-membered ring (see structure), the molecule can apparently fold into a "castanetlike" conformation trapping a molecule of ethyl acetate. ${ }^{2}$ However, this possibility remains to be conclusively determined. A full set of 5825 unique intensities to $\Theta 75^{\circ}$ was measured.

Regardless, the data greatly favor the conclusion that authentic duclauxin is produced by $P$. herquei whether grown on Gostar or enriched media. A similar anomaly regarding mp was previously reported for diplodiol (diplosporin) from Diplodia macrospora. Chalmers et al. ${ }^{23}$ reported a $\mathrm{mp}$ for diplosporin (diplodiol) of $60^{\circ} \mathrm{C}$, whereas Cutler et al. ${ }^{24}$ reported a 
mp for diplodiol of $47-48^{\circ} \mathrm{C}$. The groups exchanged samples and all other characteristics including stereochemistry, GCMS, ${ }^{1} \mathrm{H}$ NMR, ${ }^{13} \mathrm{C}$ NMR, and IR spectroscopy, and TLC values were identical. As for duclauxin, the only explanation offered for the observed anomaly was the different solvents of crystallization that were used. However, no exact resolution of the discrepancy was ever determined.

\section{References and Notes}

1. Shibata, S.; Ogihara, Y.; Tokutake, N.; Tanaka, O. Tetrahed. Lett. 1965, 18, 1287-1288.

2. Ogihara, Y.; Iitaka, Y.; Shibata, S. Acta Crystallogr. 1968, B24, 1037-1047.

3. Fuska, J.; Kuhr, I.; Nemec, P.; Fuskova, A. J. Antibiot. 1974, 27, 123-127.

4. Jong, S. C.; Gantt, M. J. ATCC Catalogue of Fungi/Yeasts, Seventeenth Edition; ATCC: Rockville, MD, 1987; p 431.

5. Wells, J. M.; Payne, J. A. Appl. Microbiol. 1975, 30, 536-540.

6. Yamazaki, M.; Okuyama, E. Chem. Pharm. Bull. 1980, 28(12), $3649-3655$.

7. Fuskova, A.; Proksa, B.; Fuska, J. Pharmazie 1977, 32, 291-293.

8. Kawai, K.; Nozawa, Y.Res. Comm. Chem. Path. Pharm. 1982, 36, $429-438$.

9. Shiojiri, H.; Kawai, K.; Kato, T.; Ogihara, Y.; Nozawa, Y. Proc. Jpn. Assoc. Mycotoxicol. 1983, 18, 38-41.

10. Kovac, L.; Bohmerova, E.; Fuska, J. J. Antibiot. 1978, 31, 616-620.

11. Kawai, K.; Shiojiri, H.; Nakamaru, T.; Nozawa, Y.; Sugie, S.; Mori, H.; Kato, T.; Ogihara, Y. Cell Biol. Toxicol. 1985, 1, 1-10.

12. Diener, U. L.; Pettit, R. E.; Cole, R. J. Peanut Science and Technology; Pattee, H. E.; Young, C. T., Eds.; American Peanut Research and Educational Society: Yoakum, TX, 1982; pp 486519.

13. Bolliger, H. R.; Brenner, M.; Ganshirt, H.; Mangold, H. K. Seiler, H.; Stahl, Egon; Waldi, D. Thin Layer Chromatography;
Stahl, E., Ed.; Springer-Verlag, Academic: New York, NY, 1965; p 486.

14. Jacyno, J. M.; Montemurro, N.; Bates, A. D.; Cutler, H. G. J. Agric. Food Chem. 1991, 39, 1166-1168.

15. Hudson, J. B.; Lopez-Bazzocchi, I.; Towers, G. H. N. Antiviral Res. 1991, 15, 101-112.

16. Kuhr, I.; Fuska, J.; Sedmera, P.; Podojil, M.; Vokoun, J.; Vanek, Z. J. Antibiot. 1973, 26, 535-536.

17. Kurtz, T. E.; Link, R. F.; Tukey, J. W. I.; Wallace, D. L. Technometric 1965, 7, 95-161.

18. Woodroof, J. G. Peanuts: production processing products; The Avi Publishing: Westport, CT, 1973; pp 292-293.

19. Treadway, R. H. Potato Processing; Talburt, W. F.; Smith, O., Eds.; Van Nostrand Reinhold: New York, NY, 1987; pp 647-663.

20. Davidson, J. I., Jr.; Whitaker, T. B.; Dickens, J. W. Peanut Science and Technology; Pattee, H. E.; Young, C. T., Eds.; American Peanut Research and Education Society: Yoakum, TX, 1982; p 608.

21. Pailthrop, R. E.; Filbert, J. W.; Richter, G. A. Potato Processing; Talburt, W. F.; Smith, O., Eds.; Van Nostrand Reinhold: New York, NY, 1982; pp 747-788.

22. Cutler, H. G. The Science of Allelopathy; Putnam, A.; Tang, C-S., Eds.; John Wiley and Sons: New York, NY, 1986; pp 147-170.

23. Chalmers, A. A.; Gorst-Allman, C. P.; Kriek, N. P. J.; Marasas, W. F. O.; Steyn, P. S.; Vleggar, R. S. Afr. J. Chem. 1978, 31, 111.

24. Cutler, H. G.; Crumley, F. G.; Cox, R. J.; Dorner, J. W.; Latterell, F. M.; Rossi, A. E. J. Agric. Food Chem. 1980, 28, 135-138.

\section{Acknowledgments}

We thank John Harwood (University of Georgia) for NMR spectra, G. H. N. Towers (University of British Columbia) for performing the antiviral testing, Bob Horvat for performing the MS, and Gary Newton for performing the X-ray diffraction. The peanut hulls were a gift from Dr. Richard Cole (USDA, ARC, National Peanut Research Laboratory, Dawson, GA, 31742). 